\title{
Analysis and solution of problems in the development of automatic color sorting systems
}

\author{
Oleg I. Katin*, Ksenia I. Goryanina, and Miroslav A. Vernezi \\ Don State Technical University, 344000 Rostov-on-Don, Russia
}

\begin{abstract}
The development of automatic sorting systems is relevant and in demand in various industries. In particular, in the food industry, such systems are used to sort different fruits of plants by color. However, there are a number of difficulties associated with the development process. These include filtering the light flux and finding the optimal data processing. This article briefly reflects the steps that have been taken in finding the best way to illuminate the control area, and describes how to solve the problem of the influence of different types of lighting on the sensor readings. The method of processing data from the color sensor by the method of standards is also considered. The described solutions allow to achieve the required results in the development or optimization of automatic color sorting systems.
\end{abstract}

\section{Introduction}

A common process in the industry is the sorting of objects on any grounds [1]. In particular, the food industry requires sorting fruits, vegetables or other fruits of plants by color. Such sorting is usually carried out on a conveyor line, along which the fruits are moving in a continuous flow. It can be both manual and automatic. Manual sorting requires several workers to visually identify the unsuitable fruit and remove it from the line. To automate the control, various hardware and software tools are used, which also allow to separate the suitable fruits from the ones unsuitable for external signs [2, 3].

Currently, automatic sorting systems are quite complex and expensive. In this regard, their share in the food industry is small. At the same time, the performance of such production systems is much higher than manual labor. Therefore, the development of such a system is a popular and urgent task.

The purpose of this article is to analyze the problems encountered in the development of automated object color sorting system and a description of ways to solve them.

\footnotetext{
* Corresponding author: okatin96@yandex.ru
} 


\section{Features of the RGB sensor}

The basis of the whole system is a color sensor. It is on its characteristics, design and method of measurement will depend on the whole system.

The module based on TCS3200 was chosen as a color sensor, an example of which is shown in Fig. 1.

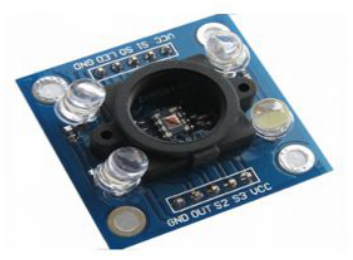

Fig. 1. The module based on TCS3200 color sensor.

It works on the principle of proportional conversion of light intensity to the output signal frequency and is a programmable set of silicon photodiodes and a voltage converter on photodiodes into a rectangular signal of a certain frequency at the output of the scheme $[4,5]$.

The sensor TCS3200, the appearance of which is shown in Fig. 2, contains 64 photodiodes arranged by a square of 8 rows and 8 columns. They can be divided into 4 groups of 16 photodiodes. The first, second and third groups have respectively light filters of certain colors: red, green, blue. Filters are not installed on photodiodes of the fourth group. Only one group of photodiodes can be measured at a time.

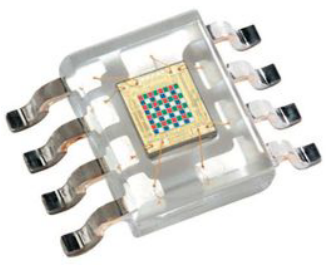

Fig. 2. The color sensor TCS3200.

This design assumes a strong dependence of the measurement results on the characteristics of light filters on photodiodes. It is also important that the sensor is sensitive not only to the visible spectrum of light. The specification contains graphics that reflect its characteristics. Also, as you can see in Fig. 3, the characteristics are uneven, resulting in greater sensitivity to red [6].

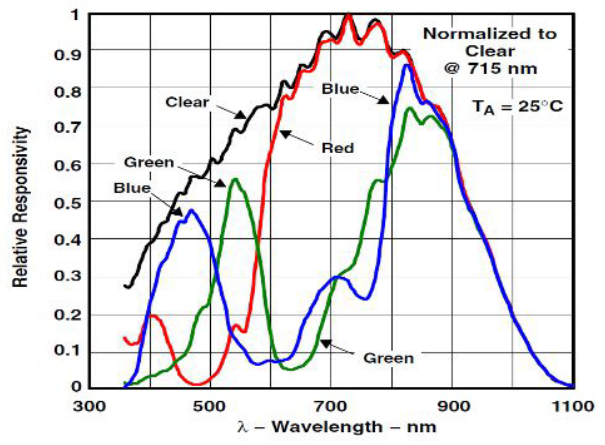

Fig. 3. Spectral sensitivity of photodiodes. 
Based on this, it can be concluded that the fundamental method will be the lighting of the object during the color determination [7].

Let us consider the spectral characteristics of some light sources [8].

The most uniform is the spectrum of daylight (Fig. 4), which favorably affects the accuracy of measurements [9-12]. However, in real conditions, such lighting can not be used, as it depends on the time of day and weather conditions.

\section{Daylight}

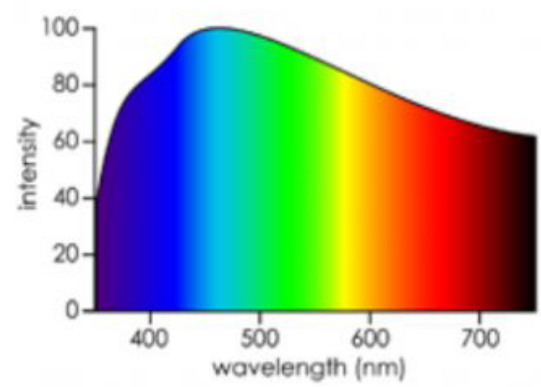

Fig. 4. Daylight spectre.

The spectrum of fluorescent lamps has pronounced peaks (Fig. 5), which, according to the test results, leads to errors in determining the color of the object [9-12].

\section{Fluorescent}

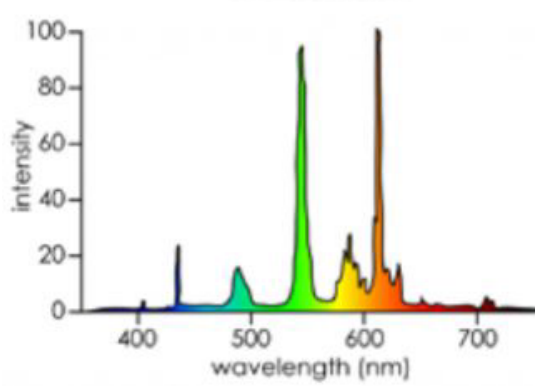

Fig. 5. Fluorescent spectre.

The most suitable lighting option for the measuring object is the LEDs of cold white light. Their spectrum allows to eliminate the factor of excessive sensitivity to red light and compensate for the weak sensitivity to blue and green (Fig. 6) [9-12]. It is desirable to place the object in the box excluding the influence of external lighting [13].

\section{Cool White LED}

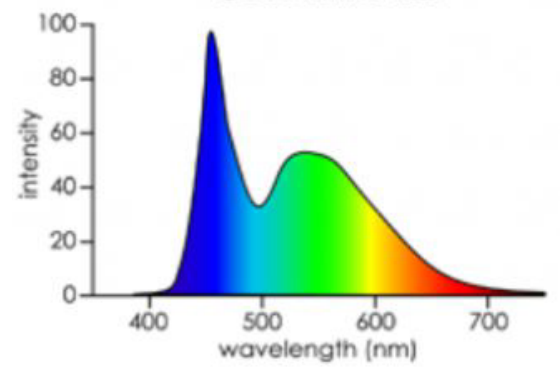

Fig. 6. Cool White LED spectre. 


\section{Processing of measurement results}

Based on the fact that the system uses RGB encoding, the measurement results can be presented in the form of a three-dimensional point cloud. To make a decision on the compliance of the object with the requirements, the method of standards is used [14].

$$
l_{i}=\left\{\sum_{j=1}^{m}\left|C_{i j} \frac{x_{j}-a_{i j}}{\sigma_{i j}}\right|^{v}\right\}^{\frac{1}{v}},
$$

where $l_{\mathrm{i}}$ - is the distance to the $\mathrm{i}$-th standard,

$\mathrm{m}$ - dimension of the coordinate space,

$\mathrm{C}_{\mathrm{ij}}$ - diagnostic coefficients,

$\mathrm{x}_{\mathrm{j}}$ - coordinate of the parameter vector,

$\mathrm{a}_{\mathrm{ij}}$ - coordinate of the $\mathrm{i}$-th diagnosis standard $\mathrm{D}_{\mathrm{i}}$,

$\sigma_{\mathrm{ij}}$ - standard deviation for $\mathrm{D}_{\mathrm{i}}$ diagnosis,

$\mathrm{V}$ - is the order of distance.

In our case, the formula is simplified, since the diagnostic coefficients for the three colors are equal. And the order of distance $v=2$, since the work with the usual distance [14] is carried out. The final form of the formula is shown below.

$$
l_{i}=\left\{\sum_{j=1}^{m}\left|x_{j}-a_{i j}\right|^{2}\right\}^{\frac{1}{2}}
$$

It is necessary to make a correction for the measurement error and slight color deviations. The result of the calculations is the $l_{i}$ vector module.

Then the area of correspondence of the object to the standard will be a sphere of radius $R$. the value of $R$ depends on the permissible color deviations and is individual for different types of objects [15].

The tests were carried out using balls of different colors with a diameter of 40 millimeters. For one run on the line in a random order was filed 25 balls, of which: 6 red were taken as the standard, 13 green, 3 blue and 3 orange. Fig. 7 shows the distribution of balls in the three-dimensional space of the RGB model.

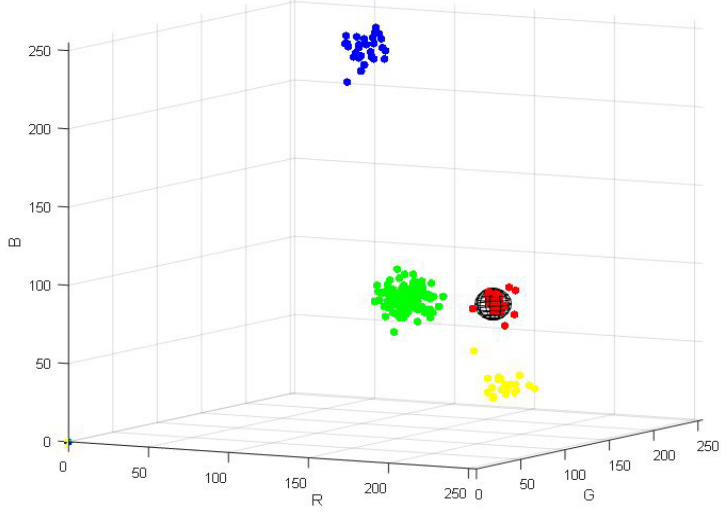

Fig. 7. The distribution of balls by color.

The points are located in the space with the RGB coordinate system of the model. The intensity of the primary colors varies from 0 to 255 . At the same time, the graph clearly shows four areas in which all the points are concentrated. Three of them correspond to 
green, blue and orange balls, and the fourth - red, it is limited to the sphere. If a point falls into an area bounded by a transparent sphere, the ball is considered to meet the standard. The size of the bounding sphere is determined based on the permissible color deviations of the objects.

\section{Conclusion}

Different lighting options have unique spectral characteristics. However, the best option should be based on the characteristics of the sensor. This relationship allows you to neutralize the disadvantages of color sensor filters by using cold white light LEDs.

There may also be different ways to process sensor data. In the course of the study, preference was given to the method of standards, as it provides the necessary accuracy and does not require complex calculations, which favorably affects the speed of the system. This result is achieved by simplifying the formula and minor mathematical assumptions.

\section{References}

1. A.V. Provotorov, System analysis in science and education, 1, 85 (in Russian) (2011)

2. O. I. Katin, K. I. Goryanina, Innovative engineering in the formation of investment attractiveness of the region: collection of scientific works. labour. II open international. scientific.- prakt. forum's, 348 (in Russian) (2017)

3. P. A. Burdovitsin, Journal of science and creativity, (in Russian), 12, 30 (2016)

4. O. I. Katin, A. D. Lukyanov, The Condition and prospects of development of agricultural mechanical engineering: collection of articles of the $\mathrm{X}$ Intern. scientific practice. Conf., 604 (2017)

5. G. Held, Introduction to Light Emitting Diode Technology and Applications, CRC Press (Worldwide, 2008)

6. TCS3200, TCS3210 Programmable color light - to-frequency converter-TAOS Inc., July 2009-access URL: http://www.datasheetlib.com/datasheet/ 1131408 / tcs3200-dtr_ams-austriamicrosystems.html

7. G. Brooker, Introduction to Sensors for Ranging and Imaging (ScitTech Publishing, 2009)

8. T. I. Trofimova, Course of physics: textbook for universities (in Russian) (PC "Academy", Moscow, 2004)

9. B. A. Shenoi, Introduction to digital signal processing and filter design (John Wiley \& Sons, Hoboken, 2006)

10. O. Katin, A. Lukyanov, K. Goryanina, MATEC Web Conf., 132, 04010 (2017)

11. A. B. Sergienko, Digital signal processing (in Russian) (Peter, Saint Petersburg, 2002)

12. K. Rauscher, F. Janssen, R. Meinhold, Fundamentals of spectrum analysis (HotlineTelecom, Moscow, 2006)

13. C. K. Madsen, J. H. Zhao, Optical Filter Design and Anal-ysis: A Signal Processing Approach (John Wiley \& Sons, New York, 1999)

14. E. R. Goryainov, A. R. Pankov, E. N. Platonov, Applied methods of statistical data analysis: proc. the allowance (in Russian) (Ed. house of Higher school of Economics, Moscow, 2012)

15. G. S. Warren, Algorithmic tricks for programmers (in Russian) (Williams, Moscow, 2014) 